\title{
Uniaxially crumpled graphene as a platform for guided myotube formation
}

\author{
Junghoon Kim', Juyoung Leem (1)', Hong Nam Kim², ${ }^{2}$, Pilgyu Kang ${ }^{1,4}$, Jonghyun Choi ${ }^{1}$, Md Farhadul Haque \\ Daeshik Kang $\mathbb{e}^{5}$ and SungWoo Nam (1) ${ }^{1,6}$
}

\begin{abstract}
Graphene, owing to its inherent chemical inertness, biocompatibility, and mechanical flexibility, has great potential in guiding cell behaviors such as adhesion and differentiation. However, due to the two-dimensional (2D) nature of graphene, the microfabrication of graphene into micro/nanoscale patterns has been widely adopted for guiding cellular assembly. In this study, we report crumpled graphene, i.e., monolithically defined graphene with a nanoscale wavy surface texture, as a tissue engineering platform that can efficiently promote aligned C2C12 mouse myoblast cell differentiation. We imparted out-of-plane, nanoscale crumpled morphologies to flat graphene via compressive straininduced deformation. When C2C12 mouse myoblast cells were seeded on the uniaxially crumpled graphene, not only were the alignment and elongation promoted at a single-cell level but also the differentiation and maturation of myotubes were enhanced compared to that on flat graphene. These results demonstrate the utility of the crumpled graphene platform for tissue engineering and regenerative medicine for skeletal muscle tissues.
\end{abstract}

\section{Introduction}

Native human tissues possess inherent anatomical directionality, and such directionality endows tissuespecific functions ${ }^{1}$. For example, skeletal muscles have longitudinally aligned myofibrils presenting directional contractile functions in response to stimuli ${ }^{2}$. Ligaments and tendons have unidirectionally aligned collagen fibers and thus absorb sudden mechanical forces by the shearing mechanism of the protein matrix ${ }^{3}$. In addition to those muscular tissues, the hippocampus in brain tissue has directionally sprouting neuronal networks, allowing a unidirectional signal transfer through the CA1-CA3 neural circuit $^{4}$. Because geometrical directionality has a close relationship with function, the recapitulation of anisotropic

\footnotetext{
Correspondence: Daeshik Kang (dskang@ajou.ac.kr) or SungWoo Nam (swnam@illinois.edu)

${ }^{1}$ Department of Mechanical Science and Engineering, University of Illinois at Urbana-Champaign, Urbana, IL 61801, USA

${ }^{2}$ Center for BioMicrosystems, Brain Science Institute, Korea Institute of Science and Technology (KIST), Seoul 02792, Republic of Korea

Full list of author information is available at the end of the article. These authors contributed equally: Junghoon Kim, Juyoung Leem, Hong Nam Kim
}

orientation has been conceived as a key factor in the fabrication of in vitro tissue models ${ }^{5,6}$. One simple yet robust method is the fabrication of anisotropic structures and the seeding of tissue-specific cells on top of tissue scaffolds. Using this method, cells recognize the orientation and surface materials of the underlying substrates and adapt their behaviors based on physicochemical cues. To prepare directional micro/nanostructures, various microfabrication methods, such as photolithography, soft lithography, and electrospinning, have been developed ${ }^{7-9}$.

In the fabrication of in vitro tissue models, the material properties are also an important issue. Skeletal muscle, heart, and brain tissues have electrical stimuli-responsive characteristics. The introduction of cyclic electrical stimuli during the culture period can promote the differentiation and functional maturation of $\mathrm{C} 2 \mathrm{C} 12$ mouse myoblast cells ${ }^{10-12}$. Even in the absence of electrical stimuli, simply cultivating $\mathrm{C} 2 \mathrm{C} 12$ mouse myoblast cells and neurons on conductive materials enhances differentiation $^{13}$. These results clearly show the importance of the electrically conductive nature of the matrix in the development of electrically active tissue models. In this regard,

\section{(c) The Author(s) 2019}

(c) (i) Open Access This article is licensed under a Creative Commons Attribution 4.0 International License, which permits use, sharing, adaptation, distribution and reproduction cc) in any medium or format, as long as you give appropriate credit to the original author(s) and the source, provide a link to the Creative Commons license, and indicate if changes were made. The images or other third party material in this article are included in the article's Creative Commons license, unless indicated otherwise in a credit line to the material. If material is not included in the article's Creative Commons license and your intended use is not permitted by statutory regulation or exceeds the permitted use, you will need to obtain permission directly from the copyright holder. To view a copy of this license, visit http://creativecommons.org/licenses/by/4.0/. 
graphene has substantial potential in the recapitulation of physiological electrical properties because it is a very thin material with high electrical conductivity ${ }^{14}$. Furthermore, graphene shows promise in the regulation of cell functions, such as promoting cell adhesion and enhancing stem cell differentiation ${ }^{15-17}$ because the adhesion of growth factors on a graphene film is promoted by interactions with $\pi-\pi$ stacking $^{18}$ and enhanced grapheneplasma membrane interaction, which regulates downstream cell signaling pathways ${ }^{19}$.

To utilize the characteristics and topographical effect of graphene in tissue engineering, a variety of approaches have been introduced. As a straightforward approach, rectangular graphene patterns with a large area of $150 \times$ $1500 \mu \mathrm{m}$ were fabricated using standard photolithography methods, and myogenic differentiation of $\mathrm{C} 2 \mathrm{C} 12$ mouse myoblast cells was demonstrated on the patterns ${ }^{20}$. As a different approach, a suspended graphene sheet was fabricated by transferring a graphene film on top of engineered nanogrooves ${ }^{21}$. On the graphene film, human mesenchymal stem cells showed an aligned morphology due to the nanogrooves beneath the graphene film, and the morphology promoted neurogenic and osteogenic differentiation. The transfer printing of graphene oxide flakes on the substrate allowed the fabrication of strip and grid graphene oxide patterns and demonstrated the graphene-specific adhesion and controlled differentiation of adipose-derived stem cells on the graphene patterned substrates $^{22}$. A graphene-nanofiber hybrid scaffold created by electrospinning polycaprolactone nanofibers on a graphene oxide film was used to explore the potential of this material in oligodendrocyte differentiation ${ }^{23}$. Micrometer-scale graphene oxide wrinkles fabricated by an unconventional fabrication method were shown to be useful in their system in guiding cell alignment ${ }^{24}$. A microgroove-patterned methacrylated gelatin substrate showed enhanced differentiation capabilities compared to a nonpatterned methacrylated gelatin substrate ${ }^{25}$. Moreover, recent research has demonstrated that conductive three-dimensional graphene foam bioscaffolds improve the growth and differentiation of $\mathrm{C} 2 \mathrm{C} 12$ myoblasts into functional myotubes ${ }^{26}$. Although previous approaches showed the potential of graphene-based tissue engineering platforms, they usually adapted complex and multistep fabrication techniques for topographically patterned graphene substrates. To utilize novel material properties in tissue engineering applications, a simpler yet robust fabrication method is required.

In this study, we report a tissue engineering platform that can utilize the topographical features and material properties of graphene by inducing mechanical instability in a graphene film. For this purpose, we transferred graphene onto a prestretched elastomer film and subsequently released the stretch so that the film could spontaneously form anisotropic geometrical features via wrinkling phenomena. We then cultured $\mathrm{C} 2 \mathrm{C} 12$ mouse myoblast cells on the crumpled graphene and observed myotube formation and maturation in response to the anisotropic geometrical features. On the crumpled graphene substrate, $\mathrm{C} 2 \mathrm{C} 12$ myoblasts showed a highly aligned morphology following the wrinkled structures and exhibited enhanced myotube formation compared to those on the flat graphene substrate.

\section{Results and discussion}

\section{Fabrication and characterization of crumpled graphene}

To fabricate crumpled graphene structures, we transferred chemical vapor deposition (CVD)-grown graphene onto a prestretched elastomeric substrate (VHB tape) using a polydimethylsiloxane (PDMS) block as a temporary stamp. The stretchable substrate was prestretched by different amounts in the $x$-direction and $y$-direction depending on the orientation to induce anisotropic mechanical instability. The major axis was stretched by $150-300 \%$, while the minor axis was stretched by $50 \%$ (Fig. 1a). We applied a stretch of $50 \%$ in the minor axis direction to compensate for the Poisson tension during the release of the stretchable substrate. By releasing the graphene-stretchable substrate complex, the stiff-soft bilayer structure forms crumpled structures due to the mismatch of the stiffness of graphene (elastic modulus $\sim 1 \mathrm{TPa})^{27,28}$ and the stretchable substrate (elastic modulus $\sim 0.59 \mathrm{MPa}$, Fig. S1) (Fig. 1a). As shown in Fig. 1 and $\mathrm{S} 2-\mathrm{S} 4$, the graphene spontaneously formed an anisotropic, nanoscale, wave-like structure without notable mechanical fractures. The k-vector of the crumpled graphene was parallel to the major axis direction. The wavelength of the crumpled graphene decreased as the prestrain increased (i.e., $c a .260 \mathrm{~nm}, 150 \mathrm{~nm}$, and $80 \mathrm{~nm}$ for 50,150 , and $300 \%$ prestrain, respectively), whereas the amplitude (displayed as the root mean square (RMS) height) increased in response to increased prestrain (i.e., ca. $8 \mathrm{~nm}, 12 \mathrm{~nm}$, and $46 \mathrm{~nm}$ for 50,150 , and $300 \%$ prestrain, respectively) (Fig. 1b; Table S1 and S2). Representative scanning electron microscopy (SEM) images show the structures of crumpled graphene in the cases of 150 and 300\% prestrain (Fig. 1d, e). Raman spectroscopy further revealed that the crumpling did not induce any permanent damage to the graphene film; the D band $\left(\sim 1350 \mathrm{~cm}^{-1}\right)$ intensity remained constant as the prestrain increased (Fig. 1c). Furthermore, the intensities of the Raman peaks from graphene increased as the prestrain increased, which indicates that the graphene wrinkles were densified with a larger prestrain.

\section{Differentiation of skeletal muscle cells}

To demonstrate the utility of crumpled graphene in skeletal muscle tissue engineering, $\mathrm{C} 2 \mathrm{C} 12$ mouse myoblast 

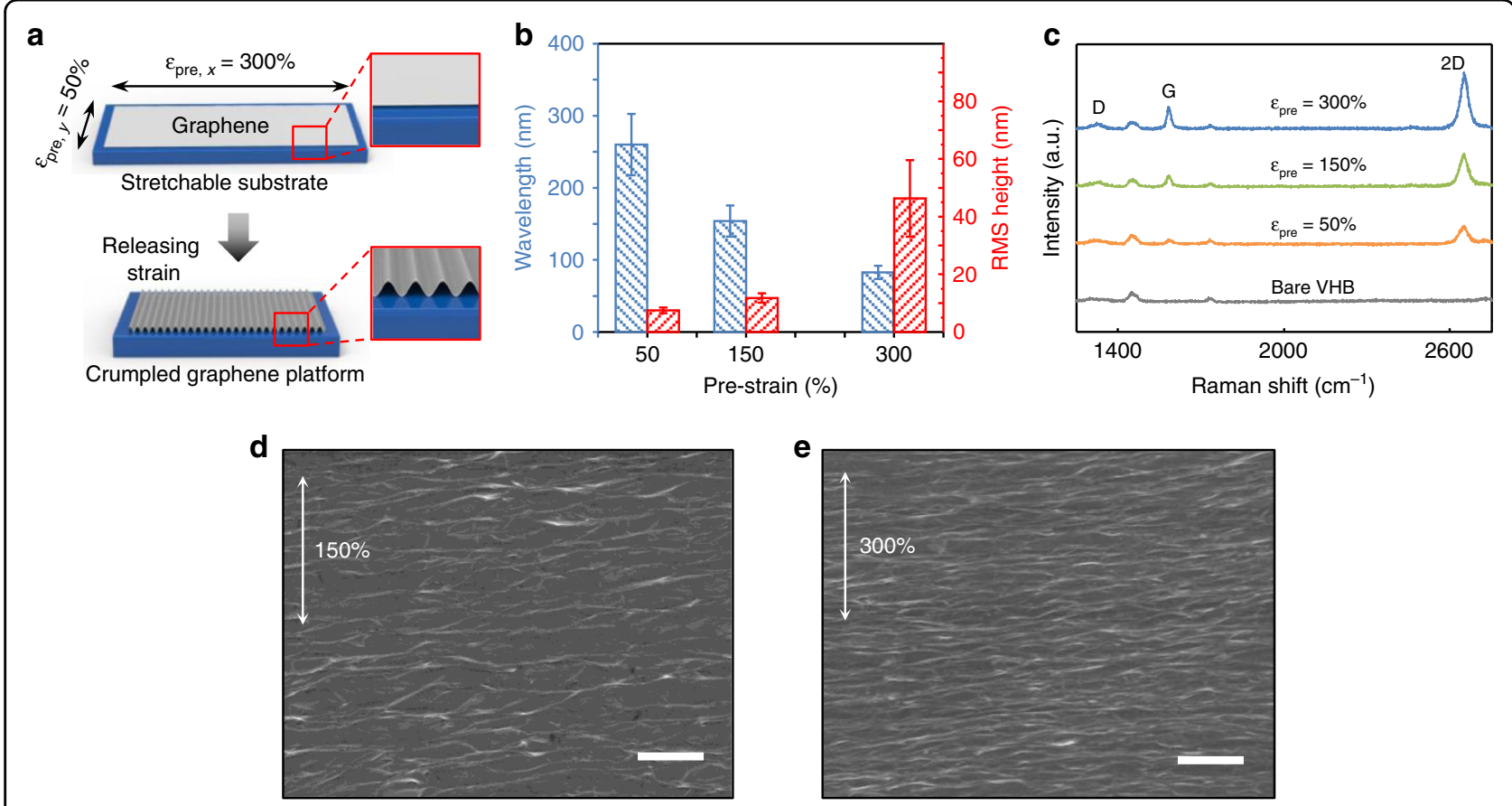

f
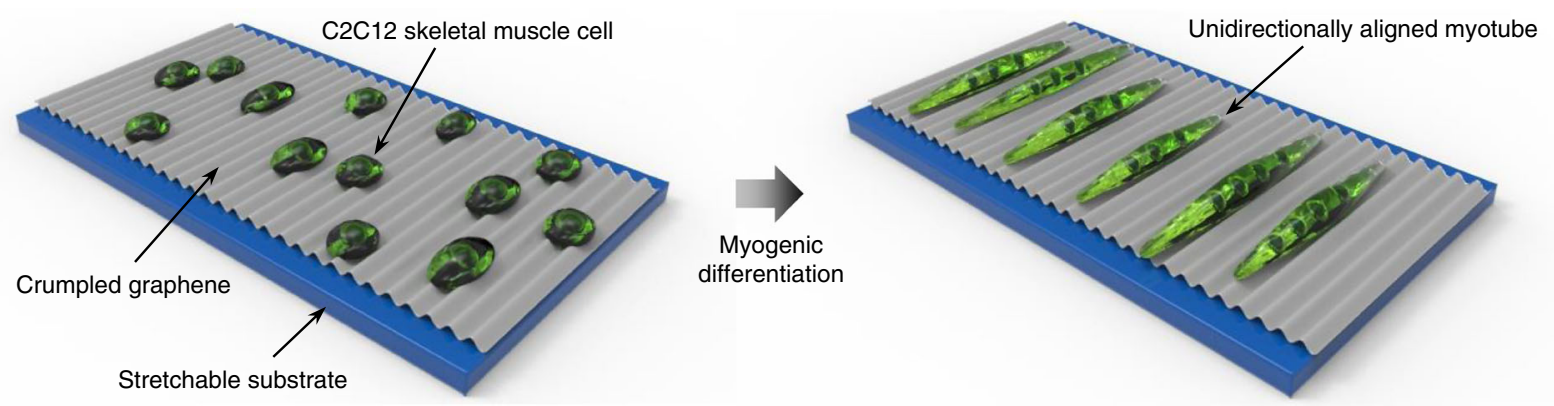

Fig. 1 Fabrication and characterization of the anisotropically crumpled graphene platform. a Fabrication of the crumpled graphene. By releasing the elastomeric substrate on which a graphene film was attached, unidirectionally crumpled graphene substrates were fabricated. b Wavelength and RMS height of crumpled graphene substrates with respect to prestrain. Previous results support that our level of imperfection (i.e., standard deviation of wavelength/height) is less than a length that a cell can recognize ${ }^{29}$. $\mathbf{c}$ Raman spectroscopy of the crumpled graphene on VHB substrates with varying prestrain and that of the bare VHB film. d-e Representative SEM images of crumpled graphene fabricated from $150 \%$ (d) and $300 \%$ (e) prestrains (scale bars: $1 \mu \mathrm{m}$ ). f Schematic illustrations of the myogenic differentiation and alignment of C2C12 cells on crumpled graphene

cells were seeded on various crumpled graphene platforms (Fig. 1f). Here, the flat graphene sample was used to identify the contribution of topographical cues to the $\mathrm{C} 2 \mathrm{C} 12$ mouse myoblast cell behaviors. Three days after seeding, the cell morphologies and their alignment behaviors were quantified using fluorescence microscopy (Fig. 2). As shown in Fig. 2a, on the flat (with graphene) substrates, the $\mathrm{C} 2 \mathrm{C} 12$ cells showed a random orientation due to the absence of topographical guidance. However, the cells showed preferential alignment, with spindle-shaped morphologies, along with a crumpled topography in the case of the 150 and 300\% prestrain platforms. Such alignment phenomena of $\mathrm{C} 2 \mathrm{C} 12$ coincided with those observed in previous studies ${ }^{13,29-32}$, showing contact guidance in response to the underlying surface topography.
To compare the differences in those cell alignment behaviors, we conducted a statistical analysis (Table 1). The circular standard deviation (the standard deviation of the cell orientation angles) decreased as the prestrain increased from 150 to $300 \%$, indicating stronger topography-induced guidance effects with higher prestrain. Rao's spacing test of uniformity is generally used to demonstrate whether the angular distributions of data are significantly different among experimental conditions. A sufficiently small $p$-value indicates that the angular distribution is significantly different from the 'uniform' distribution, with given statistical reliabilities corresponding to the $p$-values ${ }^{33}$. In the 150 and $300 \%$ prestrain cases, $p$-values smaller than 0.01 indicate a significantly different distribution of angle values from the uniform distribution. 

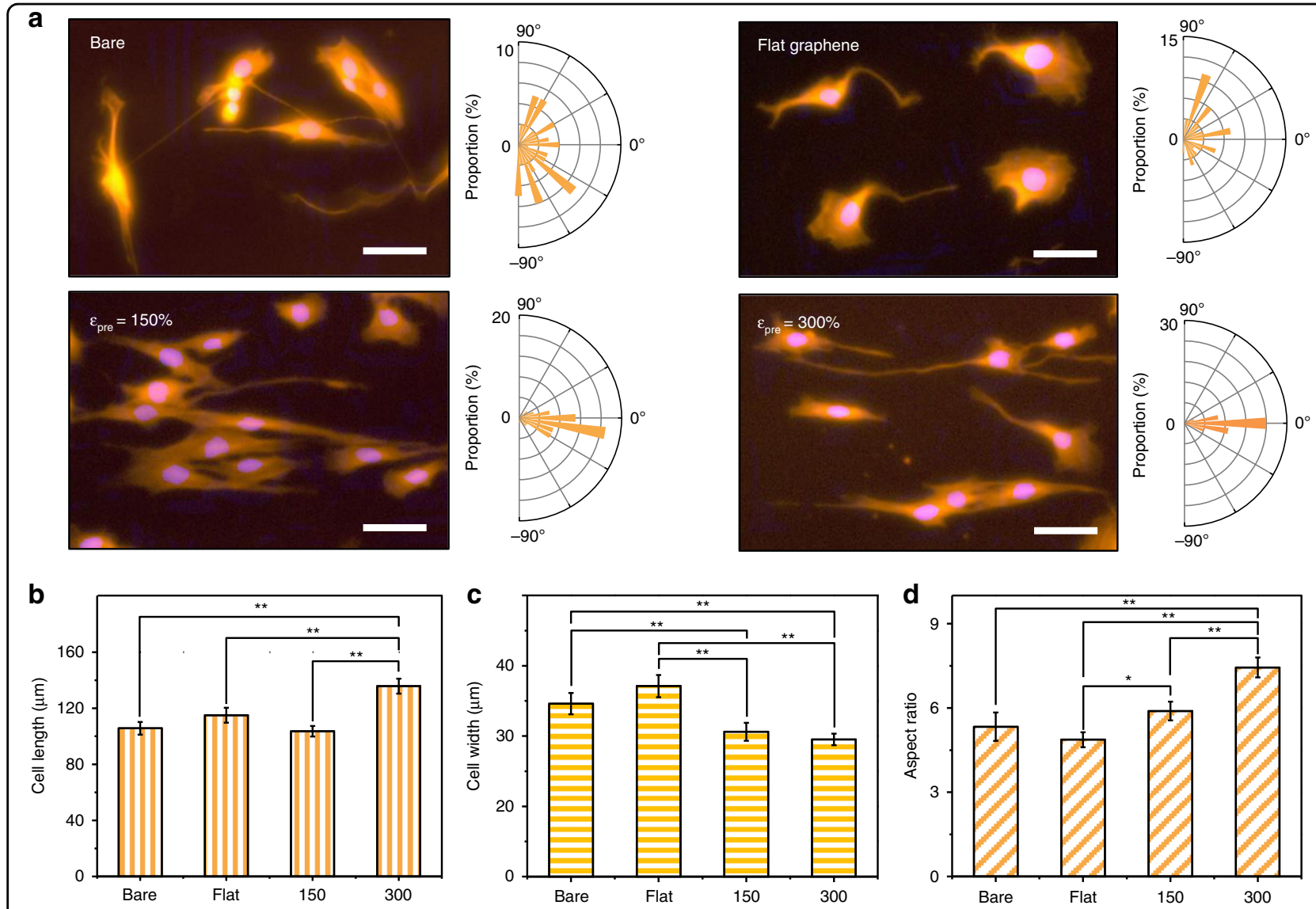

Fig. 2 Morphology of single-cell level $\mathrm{C} 2 \mathrm{C} 12$ cells 3 days after seeding on the anisotropically crumpled graphene platform. a Representative immunofluorescence images of C2C12 cells on bare (no graphene), flat (with graphene), and 150 and 300\% prestrained crumpled graphene platform (Scale bar: $50 \mu \mathrm{m}$ ). The magnification of Fig. 2a was 20x. For visualization, C2C12 mouse myoblast cells were stained with TRITCconjugated phalloidin. Red: F-actin (phalloidin), blue: nuclei (DAPI). The angular distribution of cells was quantified and visualized with the orientation rose plot. b-d Morphology analysis of C2C12 cells 3 days after seeding: (b) cell length, (c) cell width, and (d) aspect ratio (ratio of long axis to short axis). Significance: ${ }^{* *} p<0.01$ and ${ }^{*} p<0.05$. Data are represented as the mean $\pm \operatorname{SE}(n=5$, in the case of the myotube aspect ratio, $n=100)$

The Mardia-Watson-Wheeler test was further used to confirm the equality of the two angular populations ${ }^{34}$. A sufficiently small $p$-value indicates that the angular populations of two data sets are significantly different with $p$-value-specific statistical reliabilities. The Mardia-Watson-Wheeler test between the data sets, such as flat: $150 \%$ and flat: $300 \%$, showed significantly small p-values, indicating a significant difference between the cases in terms of the angular populations. These statistical analysis results consistently showed the utility of the anisotropically crumpled graphene platform for the unidirectional alignment of $\mathrm{C} 2 \mathrm{C} 12$ cells.

The morphological indices, including the cell length, cell width, and aspect ratio, were different depending on the various platforms (Fig. 2b-d). For example, C2C12 mouse myoblast cells on the $300 \%$ prestrain samples showed a much longer cell length than the other cases. Conversely, the cells on the 150 and 300\% prestrain samples displayed narrower cell widths than those on the bare and flat substrates. Due to the increased cell length and decreased cell width, the cells on the 150 and 300\% prestrain samples showed higher aspect ratios (the ratio of the long axis to the short axis), and the aspect ratio value was even larger in the $300 \%$ case than in the $150 \%$ case. The longer cell length and higher aspect ratio in the case of the $300 \%$ prestrain samples correlated with the amplitude of the crumpled graphene (Fig. 1b). Although the wavelength of the crumpled graphene decreased as the prestrain increased, the amplitude increased as the prestrain increased (Fig. 1b). This correlation between the cell morphological indices and the graphene wrinkle amplitude indicates the importance of topographical height in the guidance of cell shape and alignment. Previous studies reported that topographyinduced alignment was more effective with nanogrooves with larger height values ${ }^{35,36}$. In this regard, the higher amplitude in the case of $300 \%$ prestrain is more beneficial than that for $150 \%$ prestrain, and thus, cell alignment and elongation were promoted in the $300 \%$ prestrain case. 
Table 1 The statistical analysis of the circular standard deviation, Rao's spacing test of uniformity, and Mardia-Watson-Wheeler test for single cell-level C2C12 cells

\begin{tabular}{|c|c|c|c|c|}
\hline & Bare & Flat & $\varepsilon_{\text {pre }}=150 \%$ & $\varepsilon_{\text {pre }}=300 \%$ \\
\hline Circular standard deviation (degree) & 55.9 & 53.9 & 23.3 & 12.6 \\
\hline Rao's spacing test $(p)$ & $0.50>p>0.10$ & $0.10>p>0.05$ & $<0.01$ & $<0.01$ \\
\hline \hline Mardia-watson-wheeler test $(p)$ & Bare: $150 \%$ & $1.84 \mathrm{E}-9$ & Bare:300\% & $<1 \mathrm{E}-12$ \\
\hline
\end{tabular}
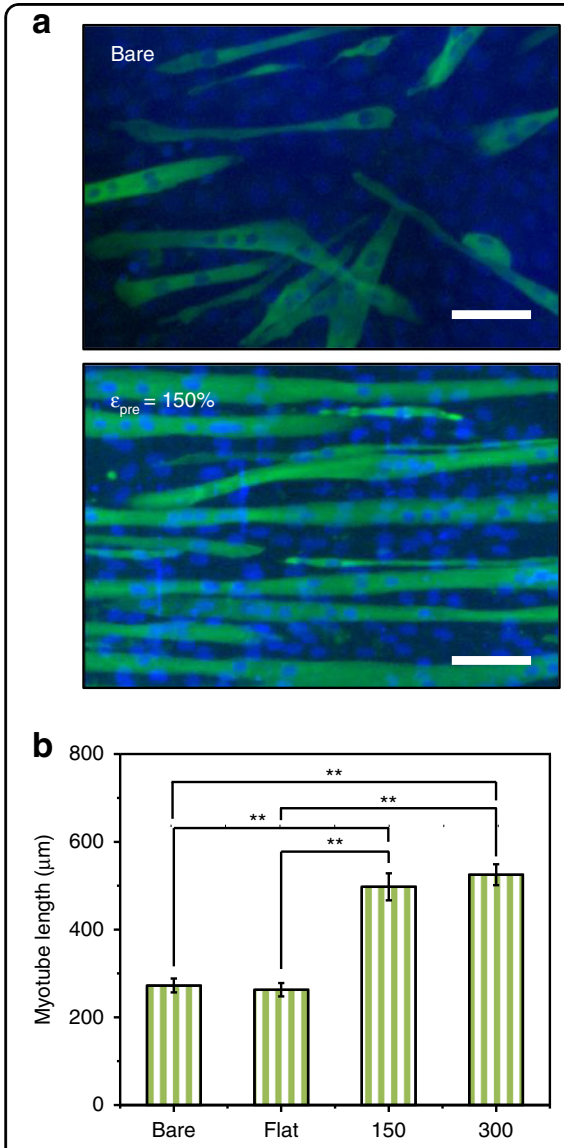
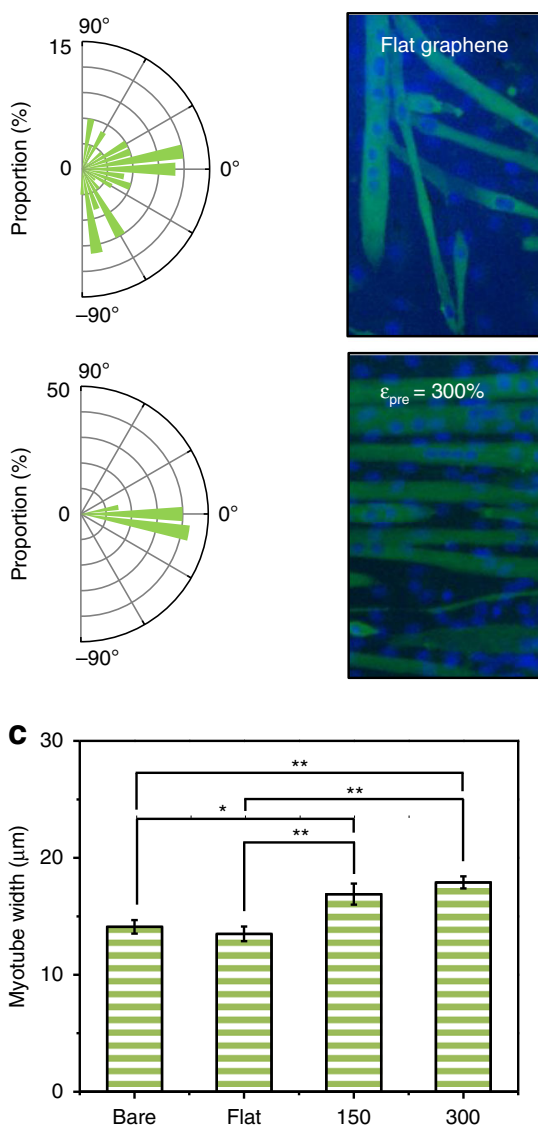
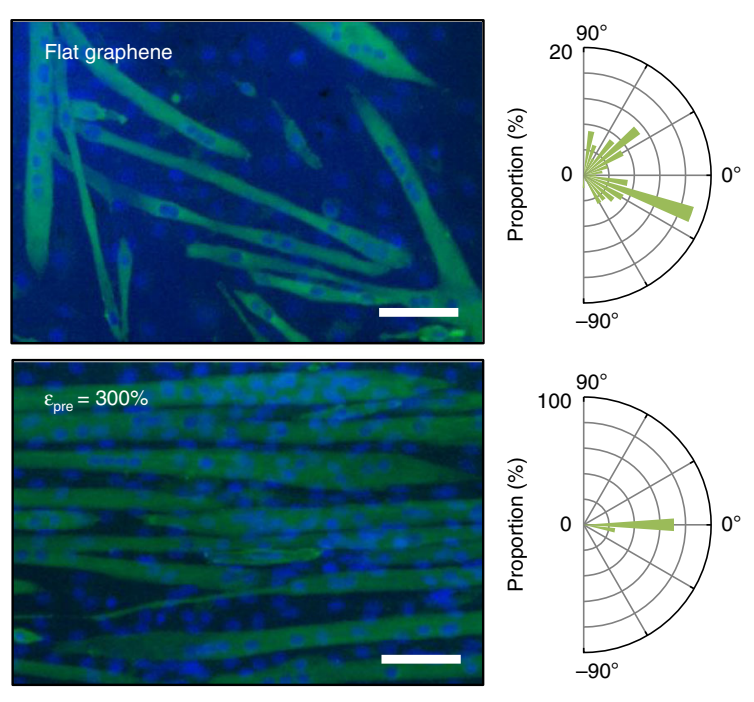

d

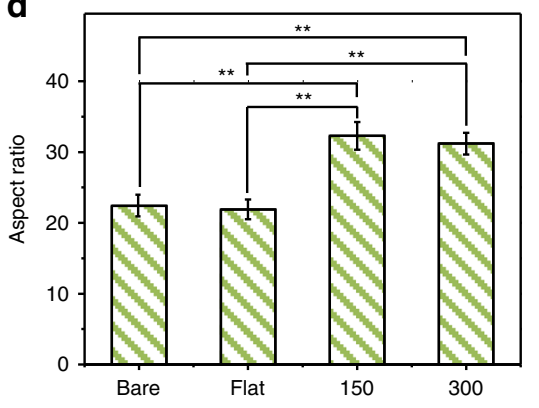

Fig. 3 Morphology of myotubes on crumpled graphene. a Representative fluorescence images and angular distribution of the myotubes on anisotropically crumpled graphene. After 7 days of differentiation of C2C12 cells in differential media, myotubes and nuclei were stained for antimyosin heavy chain (green) and DAPI (blue), respectively (scale bar: $100 \mu \mathrm{m}$ ). The magnification of Fig. 3a was 10x. b-d Quantitative analysis of myotube morphologies: (b) myotube length, (c) myotube width, and (d) aspect ratio. Significance: ${ }^{* *} p<0.01$ and ${ }^{*} p<0.05$. Data are represented as the mean $\pm \operatorname{SE}(n=5$, in the case of the myotube aspect ratio, $n=100$ )

To demonstrate the role of the crumpled graphene platform in the induction of myogenic differentiation of $\mathrm{C} 2 \mathrm{C} 12$ mouse myoblast cells, the cells were induced to be differentiated in low-serum media for 7 days. The C2C12 cells fused and subsequently formed myotubes (Fig. 3 and S5). The myotubes on the 150 and 300\% prestrain samples showed clear alignment along the crumpled orientation of the graphene film (Fig. 3a). The alignment pattern of myotubes on various samples was also quantified using statistical analysis methods (Table 2). The circular standard deviation values were significantly lower for the 150 and $300 \%$ prestrain cases than for the 
Table 2 The statistical analysis of the circular standard deviation, Rao's spacing test of uniformity, and Mardia-Watson-Wheeler test for myogenic differentiation of $\mathrm{C} 2 \mathrm{C} 12$ cells

\begin{tabular}{|c|c|c|c|c|}
\hline & Bare & Flat & $\varepsilon_{\text {pre }}=150 \%$ & $\varepsilon_{\text {pre }}=300 \%$ \\
\hline Circular standard deviation (degree) & 50.6 & 46.3 & 6.2 & 3.8 \\
\hline Rao's spacing test $(p)$ & $0.50>p>0.10$ & $<0.05$ & $<0.01$ & $<0.01$ \\
\hline \hline Mardia-watson-wheeler test $(p)$ & Bare: $150 \%$ & $<1 \mathrm{E}-12$ & Bare:300\% & $<1 \mathrm{E}-12$ \\
\hline & Flat: $150 \%$ & $<1 \mathrm{E}-12$ & Flat: $300 \%$ & $<1 \mathrm{E}-12$ \\
\hline
\end{tabular}
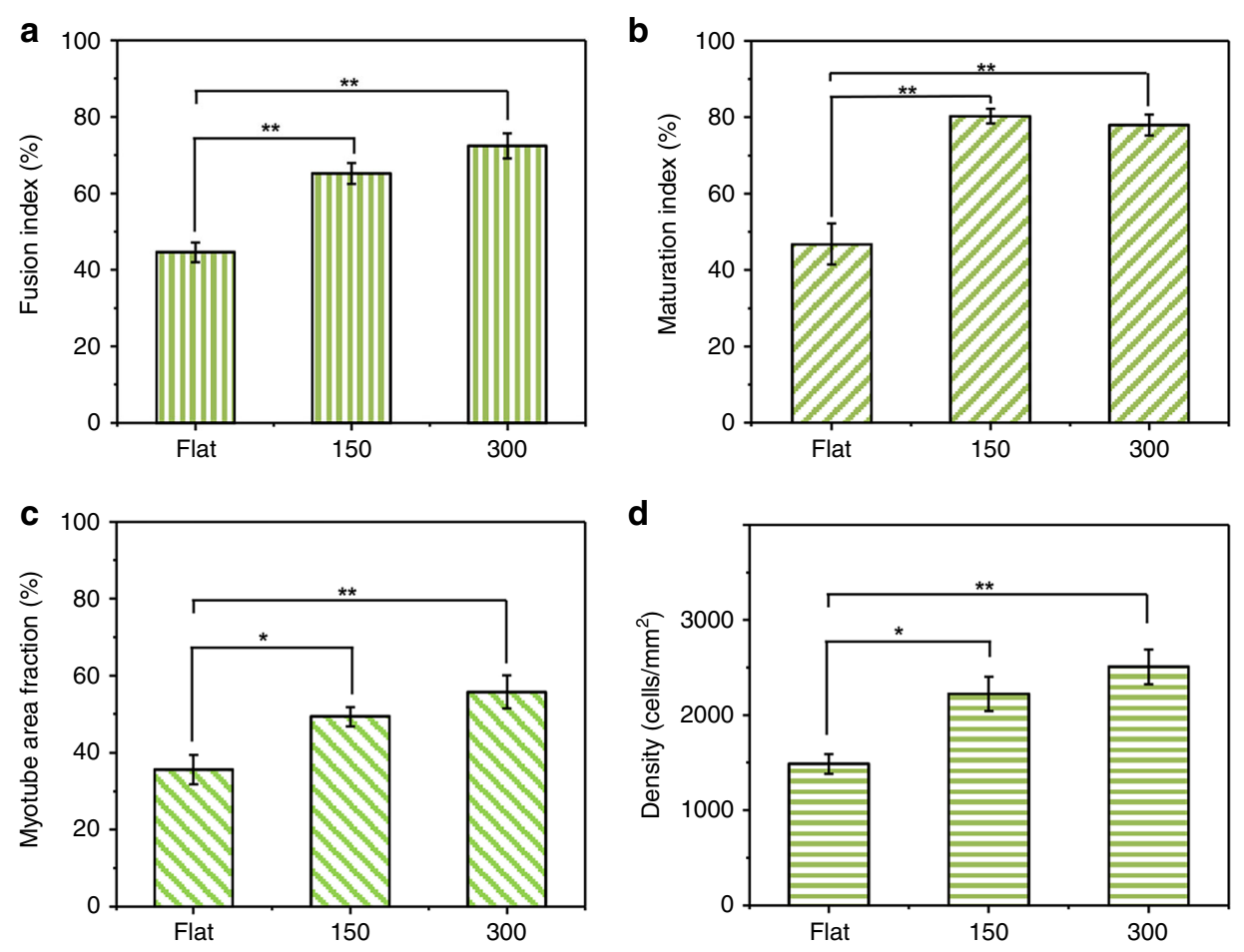

Fig. 4 Quantified maturation indices of myotubes on the anisotropically crumpled graphene platforms. a Fusion index. $\mathbf{b}$ Maturation index. $\mathbf{c}$ Myotube area fraction. $\mathbf{d}$ Density of cells on the flat/crumpled graphene. Significance: ${ }^{* *} p<0.01$ and ${ }^{*} p<0.05$. Data are represented as the mean \pm SE $(n=5$, in the case of the myotube aspect ratio, $n=100)$

flat case, and the values decreased as the prestrain value increased from 150 to $300 \%$, which is similar to the individual $\mathrm{C} 2 \mathrm{C} 12$ cell results. Rao's spacing test of uniformity showed significantly different alignment patterns of cells on the 150 and $300 \%$ samples $(p<0.01)$. The Mardia-Watson-Wheeler test demonstrated significant differences between the angular populations on various samples, such as flat:150\% and flat:300\% $\left(p<1 \mathrm{e}^{-12}\right)$. The morphological indices of myotubes were also quantified. The length, width, and aspect ratio of myotubes were larger in the case of the crumpled graphene substrates than the flat substrates (Fig. 3b-d).
However, there were no notable differences between the 150 and $300 \%$ prestrain cases.

The myogenic differentiation efficiency was evaluated with the fusion index, maturation index, myotube fraction, and myotube density (Fig. 4). The fusion index denotes the ratio of the nuclei number in myocytes with multiple nuclei, and the maturation index is the proportion of myotubes having five or more nuclei in a single myotube. The myotube area fraction is the fraction of area covered by myotubes. The density was defined as the number of nuclei within the unit area $\left(\mathrm{mm}^{2}\right)$. These four indices were higher for crumpled graphene substrates 
(150 and 300\%) than for flat substrates. Similar to the results of the morphological indices of the myotubes, there were no significant differences in those four indices between the 150 and $300 \%$ prestrain cases. According to previous studies, neither too large $(>20 \mu \mathrm{m})^{37}$ nor too small $(<70-80 \mathrm{~nm})^{29}$ size patterns affect myotube fusion, implying the limited effect of feature size control over $\mathrm{C} 2 \mathrm{C} 12$ differentiation.

To examine the change in stiffness with respect to the applied prestrain and ultimately to the cultured cell behaviors on the crumpled graphene substrates, we measured the elastic modulus of the crumpled graphene using a nanoindenter (Fig. S1). As the prestrain increased from flat to $300 \%$, the stiffness increased from $0.628 \mathrm{MPa}$ to $1.64 \mathrm{MPa}$. However, in between the 150 and $300 \%$ prestrain cases, the increase in stiffness was small from $1.43 \mathrm{MPa}$ to $1.64 \mathrm{MPa}$ for the 150 and $300 \%$ prestrain cases, respectively. Although some reports indicated that the increased stiffness of nanogrooves induced further elongation of cells along the pattern orientation ${ }^{38}$, such stiffness-dependent elongation was not observed in our study, presumably because of the small difference in stiffness.

From the quantification, we found that at the single C2C12 mouse myoblast cell level, crumpled graphene with a uniaxial topography increases the cell length while decreasing the width. Furthermore, as the prestrain increases from 150 to $300 \%$, the length and aspect ratio of the individual cells tend to increase due to the increased height of the surface texture. However, after myotube formation (i.e., myogenic differentiation), such morphological differences between the 150 and 300\% prestrain samples decreased.

Furthermore, C2C12 mouse myoblast cells and differentiated myotubes on bare VHB and flat graphene on VHB (Figs. 2-4) do not show significant differences in terms of their morphological indices. We believe that the similarity of the mechanical properties of the substrates mainly guided their similarities. Previous studies indicate that the morphology of the cultured cells is mainly controlled by the mechanical properties of the substrates, rather than the chemical moieties of the substrates ${ }^{39,40}$. As reported in previous studies ${ }^{41}$, an ultrathin membrane of a rigid material has a quite limited effect on the overall mechanical properties when layered on a soft substrate. Therefore, we believe that the similarities in the mechanical properties of VHB and graphene on VHB resulted in marginal differences in the morphological indices of $\mathrm{C} 2 \mathrm{C} 12$ mouse myoblast cells before and after differentiation.

Our crumpled graphene platform is a significant departure from previous approaches for the following reasons. First, the geometrical size (wavelength and amplitude) of our platform is much smaller than that of the platforms used in previous studies, and our platform thus can stimulate cell behaviors and functions more precisely. For example, previous studies fabricated wrinkled graphene oxide films with a wavelength and amplitude of a few micrometers to a few tens of micrometers due to the thick graphene oxide film ${ }^{31}$. However, in this study, we fabricated a nanoscale (wavelength of $c a .80 \mathrm{~nm}$ and $150 \mathrm{~nm}$; and RMS height of ca. $46 \mathrm{~nm}$ and $12 \mathrm{~nm}$; for 300 and $150 \%$ prestrain, respectively) topography using a CVD-grown monolayer graphene. Although, as shown in Fig. 1b, our crumpled graphene structures are not perfectly ordered (1-standard deviation $<50 \mathrm{~nm}$ ) compared to patterns fabricated by photolithography techniques, individual $\mathrm{C} 2 \mathrm{C} 12$ cells and differentiated myotubes displayed clear alignment and elongation in the crumpled orientation. This clear contact guidance of $\mathrm{C} 2 \mathrm{C} 12$ cells on the crumpled graphene substrate and the previous result in which cells might not recognize a sufficiently small feature size $(<70-80 \mathrm{~nm})^{29}$ support that the imperfections of crumpled graphene have a negligible effect on cell behaviors. The micron-scale topography is known to have the ability to align cells towards a topographical orientation but not to effectively guide cell differentiation because the feature size is much larger than that of focal adhesions ${ }^{42}$. Conversely, the nanoscale topography can control both alignment and differentiation because the sizes of the features and focal adhesions are comparable ${ }^{43}$. Second, although previous studies reported the superior properties of graphene in the myogenic differentiation of $\mathrm{C} 2 \mathrm{C} 12$ cells $^{20}$, the synergistic role of the surface topography and the material properties of graphene have not been clearly demonstrated.

Although the differentiation of $\mathrm{C} 2 \mathrm{C} 12$ mouse myoblast cells into myotubes is mainly discussed in this study, the proliferation and metabolism of cells will be important in future studies for the application of our crumpled graphene platform in skeletal muscle tissue engineering. According to previous studies, the morphological indices of differentiated myotubes are closely related to the gene expression of mature myogenic markers ${ }^{6,13,44}$. Such morphologycontrolled cell behaviors are also commonly observed in other cell types, such as immune cells and human mesenchymal stem cells $^{45,46}$. Therefore, we believe, although further in-depth studies are needed, that the morphology control can control the differentiation and maturation of mouse myotube cells. As reported in previous studies, culturing mouse myoblast cells (especially $\mathrm{C} 2 \mathrm{C} 12$ cells) on a nanotopography upregulates multiple genes related to myogenic differentiation, such as Myf5, MyoD, and MyoG. The enhanced gene expression of myogenic differentiation was evident irrespective of the surface characteristics, including the gelatin-coated poly (lactic-coglycolic acid) (PLGA) ${ }^{6}$, sphingosine-1-phosphateconjugated PLGA ${ }^{47}$, and gold-coated polyurethane acrylate 
(PUA) substrates ${ }^{13}$. In addition, microgroove patterns also upregulated the expression levels of focal adhesion-related, actin cytoskeleton-related, and MAPK signaling-related genes $^{48}$. Furthermore, on multiscale micro-patterns and nano-patterns, aligned nanofibers on top of microgrooves with orientations perpendicular to each other, $\mathrm{C} 2 \mathrm{C} 12$ cells preferentially aligned following the nanofibers rather than the microgrooves ${ }^{49}$. These results indicate, at least in adhesion and alignment aspects, $\mathrm{C} 2 \mathrm{C} 12$ cells prefer nanoscale patterns over microscale patterns. Based on these earlier findings, future investigations on the upregulation of various myogenic genes induced by the crumpled graphene substrate will provide further evidence of the impact of the crumpled topography on myogenic differentiation. The observation of cross-striation will also provide evidence of myotube maturation. In addition to gene expression, the effect of extracellular matrix components on differentiation and subsequent production by the cells can be studied in the future. Previous researchers reported that the laminin protein significantly promotes cell adhesion and differentiation of $\mathrm{C} 2 \mathrm{C} 12$ cells ${ }^{26,50,51}$. Furthermore, we expect that these electrically active, nanotopographic crumpled graphene substrates can electrically stimulate cells. According to previous studies, consistent electrical stimulation can enhance the differentiation and maturation of skeletal muscle cells ${ }^{25,26,52-54}$. Although, in this work, there was no significant difference in the experimental results between 'bare' and 'flat' substrates due to the absence of electrical stimulation and the similarity of the elastic moduli (Fig. S1), an evaluation of the effect of electrical stimulation on crumpled graphene would be meaningful in the future ${ }^{52}$. Finally, humanoriginating skeletal cells can be used to validate the applicability of crumpled graphene platforms for skeletal muscle tissue engineering. We believe that future studies on the above aspects may further strengthen the usefulness of crumpled graphene platforms for skeletal muscle tissue engineering.

\section{Conclusions}

In this study, we report crumpled graphene, a monolithically defined graphene with a nanoscale wavy surface texture, as a tissue engineering platform that can efficiently promote aligned $\mathrm{C} 2 \mathrm{C} 12$ mouse myoblast cell differentiation. By releasing the anisotropic prestrain of an elastomer-graphene bilayer structure, unidirectionally crumpled graphene substrates were fabricated. When C2C12 mouse myoblast cells were seeded, they showed an elongated and aligned morphology in response to the increase in prestrain. After myogenic differentiation, the myotubes had a more elongated morphology and showed better maturation on the crumpled graphene substrates than on the flat substrates. These results showed that crumpled graphene substrates with unidirectional topography can be used as an effective platform for skeletal muscle tissue engineering in terms of alignment, morphology, and maturation. Furthermore, given the simplicity of crumpled substrate fabrication and the simultaneous electrical sensing/stimulation capability of graphene, we believe that our monolithic crumpled graphene platform could allow future innovations in skeletal muscle tissue engineering.

\section{Materials and methods}

\section{Fabrication of the crumpled graphene structure on a flexible substrate}

The crumpled graphene structure was fabricated by modifying the protocols of previous studies ${ }^{55,56}$. Graphene was synthesized on a $25 \mu \mathrm{m}$-thick copper $(\mathrm{Cu})$ foil (Alfa Aesar, MA) by a chemical vapor deposition (CVD) system (Rocky Mountain Vacuum Tech Inc., CO). Under a hydrogen $\left(\mathrm{H}_{2}\right)$ gas environment $(50 \mathrm{sccm})$ at $150 \mathrm{mTorr}$, the chamber was heated to $1050{ }^{\circ} \mathrm{C}$. After annealing at $1050{ }^{\circ} \mathrm{C}$ for $2 \mathrm{~h}$, methane $\left(\mathrm{CH}_{4}\right)$ and $\mathrm{H}_{2}$ gases $\left(\mathrm{CH}_{4}\right.$ : $100 \mathrm{sccm}$ and $\mathrm{H}_{2}: 50 \mathrm{sccm}$ ) were introduced into the chamber at $520 \mathrm{mTorr}$ for $2 \mathrm{~min}$. Then, the chamber was cooled down to room temperature slowly under an argon (Ar) gas environment $(500 \mathrm{sccm})$ at $330 \mathrm{mTorr}$. After the synthesis of graphene, a polydimethylsiloxane (PDMS) block was attached on the graphene side of the $\mathrm{Cu}$ foil, and then $\mathrm{Cu}$ was etched with $1 \mathrm{M} \mathrm{FeCl}_{3}$ solution. For a flexible substrate, a very high bond (VHB) substrate ( $3 \mathrm{M}$ VHB 4910, $t=1.143 \mathrm{~mm}$ ) was used. After stretching the VHB substrate in biaxial directions, with a larger prestrain applied to one direction (e.g., $300 \%$ in the $x$ direction and $50 \%$ in the $y$ direction; $\varepsilon_{\text {pre, } x}=300 \%$ and $\varepsilon_{\text {pre, } y}=50 \%$ ) to induce uniaxial deformation, the graphene on the PDMS block was transferred onto the stretched VHB substrate. Due to the mechanical limitation of the VHB substrate, the stretching of the VHB substrate was controlled to be less than $400 \%$. After transfer, the stretched VHB substrate was released, and the crumpled graphene structure was obtained. The surface structure of the crumpled graphene was monitored with a scanning electron microscope (SEM, S-4800, Hitachi) and an atomic force microscope (AFM, MFP-3D, Asylum Research), and the integrities of the crumpled graphene at various tensile strain conditions between 0 and 300\% were evaluated with a Raman spectroscope (micro PL/Raman microscope system, Renishaw) using a $633 \mathrm{~nm}$ wavelength laser. Nanoindentation was carried out with the same AFM system used for imaging.

\section{Cell morphology and angular orientation analysis}

Three types of cell substrates were prepared: two crumpled graphene substrates $\left(\varepsilon_{\text {pre, } x}: 300\right.$ and $\left.150 \%\right)$ and a flat graphene substrate (50\% prestrain). The prepared substrates were sterilized with alcohol and ultraviolet 
(UV) light. Then, mouse skeletal myoblast $\mathrm{C} 2 \mathrm{C} 12$ cells were seeded at a density of 5000 cells $/ \mathrm{cm}^{2}$ on sterilized graphene/VHB substrates. The cells were maintained in growth media (GM, DMEM containing 10\% FBS and 1\% penicillin-streptomycin) in a humidified atmosphere with $5 \% \mathrm{CO}_{2}$ at $37^{\circ} \mathrm{C}$. The $\mathrm{GM}$ was replaced every $24 \mathrm{~h}$. After 3 days of cell culture, the morphology and angular orientation data of the cells were analyzed using cytoskeleton staining. The cells were fixed with $4 \%$ paraformaldehyde, permeabilized with $0.1 \%$ Triton X-100, and stained with TRITC-phalloidin (Sigma-Aldrich, USA) and DAPI (Thermo Fisher Scientific, USA). The actin filaments and nuclei were observed using a fluorescence microscope (IX81, Olympus). From the obtained fluorescence images, cell width, length, aspect ratio, and angular orientation data were analyzed with Image software. We analyzed 5 different samples from four kinds of cell substrates and monitored over 100 cells from each sample. We conducted Rao's spacing test to evaluate the uniform distribution of angular orientation data of cells and the Mardia-Watson-Wheeler test to evaluate the identical distribution of the angular orientation data of cells.

\section{Myogenic differentiation and alignment analysis}

Two types of crumpled graphene substrates $\left(\varepsilon_{\text {pre, } x}: 300\right.$ and $150 \%)$ and a flat graphene substrate were prepared and sterilized with alcohol and UV. Mouse skeletal myoblast $\mathrm{C} 2 \mathrm{C} 12$ cells were seeded at a density of 5000 cells $/ \mathrm{cm}^{2}$ on sterilized graphene/VHB substrates. The seeded cells were grown in $\mathrm{GM}$ at $37^{\circ} \mathrm{C}$ and $5 \% \mathrm{CO}_{2}$. GM was replaced every $24 \mathrm{~h}$. When the cells covered $80 \%$ of the substrate, GM was replaced with differential media (DM, DMEM containing $2 \%$ horse serum and $1 \%$ penicillin-streptomycin). The cells were incubated at $37^{\circ} \mathrm{C}$ and $5 \% \mathrm{CO}_{2}$, and the DM was replaced every $12 \mathrm{~h}$. After 7 days of differentiation, cells were fixed, permeabilized and blocked with $4 \%$ paraformaldehyde, $0.1 \%$ Triton X100 and $1 \%$ bovine serum albumin. For immunostaining, cells were incubated with the primary antibody MF20 (anti-myosin heavy chain (MHC)), Developmental Studies Hybridoma Bank ((DSHB), University of Iowa) overnight at $4{ }^{\circ} \mathrm{C}$, followed by incubation with the secondary antibody (fluorescein isothiocyanate (FITC)-conjugated goat anti-mouse IgG, Thermo Fisher Scientific) and DAPI (Thermo Fisher Scientific) for $2 \mathrm{~h}$ at $37^{\circ} \mathrm{C}$. Myotube formation and alignment were observed using a fluorescence microscope (IX81, Olympus) and analyzed with ImageJ software. We analyzed 5 different samples from four kinds of cell substrates and monitored over 100 myotubes from each sample. To evaluate the uniform distribution and identical distribution of myotube alignment data, Rao's spacing and Mardia-Watson-Wheeler tests were conducted. To evaluate the myogenic differentiation efficiency, we analyzed the fusion index (the ratio of the nuclei number in myocytes with two or more nuclei), maturation index (the percentage of myotubes having five or more nuclei), myotube area fraction and cell density from each sample.

\section{Statistical analysis}

All of the quantitative data are expressed as the mean \pm standard error of the mean. Statistical analysis was performed by means of one-way analysis of variance. For the determination of statistical significance, $p$-values smaller than 0.05 were considered to be significant.

\section{Acknowledgements}

S.N. gratefully acknowledges support from DTRA (HDTRA1620298), NSF (MRSEC DMR-1720633 and DMR-1708852), KRISS (KRISS-2018-GP2018-0012), ONR (N00014-17-1-2830), and NASA ECF (NNX16AR56G). D.K. acknowledges financial support from NRF (2016R1C1B1009689, 2019R1H1A1080221,

2019R1A2C1090056), MOTIE (20000512), the new faculty research fund of Ajou University, and the Ajou University research fund. Experiments were carried out in part at the Materials Research Laboratory Central Research Facilities,

Holonyak Micro and Nanotechnology Laboratory, and the Beckman Institute Imaging Technology Group at the University of Illinois at Urbana-Champaign This research was partially supported by the NSF through the University of Illinois at Urbana-Champaign Materials Research Science and Engineering Center DMR-1720633.

\section{Author details}

${ }^{1}$ Department of Mechanical Science and Engineering, University of Illinois at Urbana-Champaign, Urbana, IL 61801, USA. ${ }^{2}$ Center for BioMicrosystems, Brain Science Institute, Korea Institute of Science and Technology (KIST), Seoul 02792, Republic of Korea. ${ }^{3}$ Division of Bio-Medical Science and Technology, KIST School, Korea University of Science and Technology, Seoul 02792,

Republic of Korea. ${ }^{4}$ Department of Mechanical Engineering, George Mason University, Fairfax, VA 22030, USA. ${ }^{5}$ Department of Mechanical Engineering, Ajou University, Suwon 16499, Republic of Korea. ${ }^{6}$ Department of Materials Science and Engineering, University of Illinois at Urbana-Champaign, Urbana, IL 61801, USA

\section{Conflict of interest}

The authors declare that they have no conflict of interest.

Supplementary information accompanies this paper at https://doi.org/ 10.1038/s41378-019-0098-6.

Received: 9 March 2019 Revised: 5 July 2019 Accepted: 19 August 2019 Published online: 04 November 2019

\footnotetext{
References

1. Kim, H. N. et al. Nanotopography-guided tissue engineering and regenerative medicine. Adv. Drug Deliv. Rev. 65, 536-558 (2013).

2. Lieber, R. L. \& Lieber, R. L. Skeletal Muscle Structure, Function and Plasticity: the Physiological Basis of Rehabilitation. (Lippincott Williams \& Wilkins, 2002).

3. Ji, B. \& Gao, H. Mechanical principles of biological nanocomposites. Annu. Rev. Mater. Res. 40, 77-100 (2010).

4. Kim, S. H. et al. Anisotropically organized three-dimensional culture platform for reconstruction of a hippocampal neural network. Nat. Commun. 8, 14346 (2017).

5. Kim, D.-H. et al. Nanoscale cues regulate the structure and function of macroscopic cardiac tissue constructs. Proc. Natl Acad. Sci. USA 107, 565-570 (2010).

6. Yang, H. S. et al. Nanopatterned muscle cell patches for enhanced myogenesis and dystrophin expression in a mouse model of muscular dystrophy. Biomaterials 35, 1478-1486 (2014).
} 
7. Kim, D.-H., Lee, H., Lee, Y. K., Nam, J.-M. \& Levchenko, A. Biomimetic nanopatterns as enabling tools for analysis and control of live cells. Adv. Mater. 22 4551-4566 (2010)

8. Bae, W.-G. et al. 25th anniversary article: scalable multiscale patterned structures inspired by nature: the role of hierarchy. Adv. Mater. 26, 675-700 (2014).

9. Kim, H. N. et al. Patterning methods for polymers in cell and tissue engineering. Ann. Biomed. Eng. 40, 1339-1355 (2012).

10. Flaibani, M. et al. Muscle differentiation and myotubes alignment is influenced by micropatterned surfaces and exogenous electrical stimulation. Tissue Eng. Part A 15, 2447-2457 (2009).

11. Ito, A. et al. Induction of functional tissue-engineered skeletal muscle constructs by defined electrical stimulation. Sci. Rep. 4, 4781 (2015).

12. Park, $\mathrm{H}$. et al. Effects of electrical stimulation in $\mathrm{C} 2 \mathrm{C} 12$ muscle constructs. J. Tissue Eng. Regen. Med. 2, 279-287 (2008).

13. Yang, H. S. et al. Electroconductive nanopatterned substrates for enhanced myogenic differentiation and maturation. Adv. Healthc. Mater. 5, 137-145 (2016).

14. Tang, M. et al. Enhancement of electrical signaling in neural networks on graphene films. Biomaterials 34, 6402-6411 (2013).

15. Park, S. Y. et al. Enhanced differentiation of human neural stem cells into neurons on graphene. Adv. Mater. 23, H263-H267 (2011).

16. Kalbacova, M., Broz, A., Kong, J. \& Kalbac, M. Graphene substrates promote adherence of human osteoblasts and mesenchymal stromal cells. Carbon NY 48, 4323-4329 (2010).

17. Nayak, T. R. et al. Graphene for controlled and accelerated osteogenic differentiation of human mesenchymal stem cells. ACS Nano 5, 4670-4678 (2011)

18. Kenry, Lee, W. C., Loh, K. P. \& Lim, C. T. When stem cells meet graphene: Opportunities and challenges in regenerative medicine. Biomaterials $\mathbf{1 5 5}$ 236-250 (2018).

19. Zhang, B., Wei, P., Zhou, Z. \& Wei, T. Interactions of graphene with mammalian cells: Molecular mechanisms and biomedical insights. Adv. Drug Deliv. Rev. 105, 145-162 (2016)

20. Bajaj, P., Rivera, J. A., Marchwiany, D., Solovyeva, V. \& Bashir, R. Graphene-based patterning and differentiation of C2C12 myoblasts. Adv. Healthc. Mater. 3, 995-1000 (2014).

21. Kim, J. et al. Engineering structures and functions of mesenchymal stem cells by suspended large-area graphene nanopatterns. 2D Mater. 3 , 035013 (2016).

22. Kim, T.-H. et al. Controlling differentiation of adipose-derived stem cells using combinatorial graphene hybrid-pattern arrays. ACS Nano 9 3780-3790 (2015).

23. Shah, S. et al. Guiding stem cell differentiation into oligodendrocytes using graphene-nanofiber hybrid scaffolds. Adv. Mater. 26, 3673-3680 (2014).

24. Wang, Z. et al. Wrinkled, wavelength-tunable graphene-based surface topographies for directing cell alignment and morphology. Carbon NY 97, 14-24 (2016).

25. Hosseini, V. et al. Engineered contractile skeletal muscle tissue on a microgrooved methacrylated gelatin substrate. Tissue Eng. Part A 18, 2453-2465 (2012).

26. Krueger, E. et al. Graphene foam as a three-dimensional platform for myotube growth. ACS Biomater. Sci. Eng. 2, 1234-1241 (2016).

27. Lee, C., Wei, X., Kysar, J. W. \& Hone, J. Measurement of the elastic properties and intrinsic strength of monolayer graphene. Science 321, 385-388 (2008).

28. Lee, J.-U., Yoon, D. \& Cheong, H. Estimation of Young's modulus of graphene by Raman spectroscopy. Nano Lett. 12, 4444-4448 (2012).

29. van Delft, F. C. M. J. M. et al. Manufacturing substrate nano-grooves for studying cell alignment and adhesion. Microelectron. Eng. 85, 1362-1366 (2008).

30. Kim, M. S. et al. 3D tissue formation by stacking detachable cell sheets formed on nanofiber mesh. Biofabrication 9, 015029 (2017).

31. Bettadapur, A. et al. Prolonged culture of aligned skeletal myotubes on micromolded gelatin hydrogels. Sci. Rep. 6, 28855 (2016).

32. Anene-Nzelu, C. G. et al. Scalable alignment of three-dimensional cellular constructs in a microfluidic chip. Lab Chip 13, 4124 (2013).
33. Batschelet, E. Circular Statistics In Biology. (Academic Press, London; New York 1981).

34. Li, G. N. \& Hoffman-Kim, D. Evaluation of neurite outgrowth anisotropy using a novel application of circular analysis. J. Neurosci. Methods $\mathbf{1 7 4}$ 202-214 (2008).

35. Loesberg, W. et al. The threshold at which substrate nanogroove dimensions may influence fibroblast alignment and adhesion. Biomaterials 28, 3944-3951 (2007).

36. Rajnicek, A., Britland, S. \& McCaig, C. Contact guidance of CNS neurites on grooved quartz: influence of groove dimensions, neuronal age and cell type. J. Cell Sci. 110(Pt 23), 2905-2913 (1997).

37. Bajaj, P. et al. Patterning the differentiation of C2C12 skeletal myoblasts. Integr. Biol. 3, 897 (2011)

38. Park, J., Kim, H.-N., Kim, D.-H., Levchenko, A. \& Suh, K.-Y. Quantitative analysis of the combined effect of substrate rigidity and topographic guidance on cell morphology. IEEE Trans. Nanobioscience 11, 28-36 (2012).

39. Engler, A. J. et al. Myotubes differentiate optimally on substrates with tissuelike stiffness. J. Cell Biol. 166, 877-887 (2004).

40. Yeung, T. et al. Effects of substrate stiffness on cell morphology, cytoskeletal structure, and adhesion. Cell Motil. Cytoskeleton 60, 24-34 (2005).

41. Cavallo, F., Grierson, D. S., Turner, K. T. \& Lagally, M. G. "Soft Si": effective stiffness of supported crystalline nanomembranes. ACS Nano 5, 5400-5407 (2011).

42. Kim, H. N. \& Kim, J. Effect of topographical feature size on the trend of cell behaviors. IEEE Trans. Nanotechnol. 17, 377-380 (2018).

43. Nguyen, A. T., Sathe, S. R. \& Yim, E. K. F. From nano to micro: topographical scale and its impact on cell adhesion, morphology and contact guidance. J. Phys. Condens. Matter 28, 183001 (2016).

44. Tay, C. Y. et al. Micropatterned matrix directs differentiation of human mesenchymal stem cells towards myocardial lineage. Exp. Cell Res. 316, 1159-1168 (2010).

45. McWhorter, F. Y., Wang, T., Nguyen, P., Chung, T. \& Liu, W. F. Modulation of macrophage phenotype by cell shape. Proc. Natl Acad. Sci. 110, 17253-17258 (2013).

46. Kim, J. et al. Designing nanotopographical density of extracellular matrix for controlled morphology and function of human mesenchymal stem cells. Sci. Rep. 3, 3552 (2013).

47. Tsui, J. H. et al. Harnessing sphingosine-1-phosphate signaling and nanotopographical cues to regulate skeletal muscle maturation and vascularization. ACS Nano 11, 11954-11968 (2017).

48. Gao, H., Cao, X., Dong, H. \& Fu, X. Influence of 3D microgrooves on C2C12 cell proliferation, migration, alignment, F-actin protein expression and gene expression. J. Mater. Sci. Technol. 32, 901-908 (2016).

49. Cha, S. H., Lee, H. J. \& Koh, W.-G. Study of myoblast differentiation using multidimensional scaffolds consisting of nano and micropatterns. Biomater. Res. 21, 1 (2017).

50. Parker, F., White, K., Phillips, S. \& Peckham, M. Promoting differentiation of cultured myoblasts using biomimetic surfaces that present alpha-laminin-2 peptides. Cytotechnology 68, 2159-2169 (2016).

51. Schuler, F. \& Sorokin, L. M. Expression of laminin isoforms in mouse myogenic cells in vitro and in vivo. J. Cell Sci. 108(Pt 12), 3795-3805 (1995).

52. Zhang, Z., Klausen, L. H., Chen, M. \& Dong, M. Electroactive scaffolds for neurogenesis and myogenesis: graphene-based nanomaterials. Small $\mathbf{1 4}$ 1801983 (2018).

53. Ahadian, $\mathrm{S}$. et al. Electrically regulated differentiation of skeletal muscle cells on ultrathin graphene-based films. RSC Adv. 4, 9534 (2014).

54. Burch, N. et al. Electric pulse stimulation of cultured murine muscle cells reproduces gene expression changes of trained mouse muscle. PLOS ONE $\mathbf{5}$ e10970 (2010).

55. Zang, J. et al. Multifunctionality and control of the crumpling and unfolding of large-area graphene. Nat. Mater. 12, 321-325 (2013).

56. Wang, M. C. et al. Heterogeneous, three-dimensional texturing of graphene Nano Lett. 15, 1829-1835 (2015). 